\title{
Velocity-metallicity correlation for high-z DLA galaxies: evidence of a mass-metallicity relation? ${ }^{\star}$
}

\author{
C. Ledoux ${ }^{1}$, P. Petitjean ${ }^{2,3}$, J. P. U. Fynbo ${ }^{4}$, P. Møller ${ }^{5}$, and R. Srianand ${ }^{6}$ \\ 1 European Southern Observatory, Alonso de Córdova 3107, Casilla 19001, Vitacura, Santiago 19, Chile \\ e-mail: cledoux@eso.org \\ 2 Institut d'Astrophysique de Paris - UMR 7095 CNRS \& Université Pierre et Marie Curie, 98bis Boulevard Arago, 75014 Paris, \\ France \\ e-mail: petitjean@iap.fr \\ 3 LERMA, Observatoire de Paris, 61 Avenue de l'Observatoire, 75014 Paris, France \\ 4 Dark Cosmology Centre, Niels Bohr Institute, University of Copenhagen, Juliane Maries Vej 30, 2100 Copenhagen $\emptyset$, Denmark \\ e-mail: jfynbo@astro.ku.dk \\ 5 European Southern Observatory, Karl-Schwarzschild-Straße 2, 85748 Garching bei München, Germany \\ e-mail: pmoller@eso.org \\ 6 Inter-University Centre for Astronomy and Astrophysics, Post Bag 4, Ganesh Khind, Pune 411 007, India \\ e-mail: anand@iucaa.ernet.in
}

Received 23 September 2005 / Accepted 22 May 2006

\section{ABSTRACT}

\begin{abstract}
We used our database of VLT-UVES quasar spectra to build up a sample of 70 Damped Lyman- $\alpha$ (DLA) or strong sub-DLA systems with total neutral hydrogen column densities of $\log N(\mathrm{HI}) \gtrsim 20$ and redshifts in the range $1.7<z_{\mathrm{abs}}<4.3$. For each of the systems, we measured the metallicities relative to solar in an homogeneous manner, $[\mathrm{X} / \mathrm{H}]$ (with $\mathrm{X}=\mathrm{Zn}$, or S or Si), and the velocity widths of lowionization line profiles, $\Delta V$. For the first time, we provide evidence for a correlation between DLA metallicity and line profile velocity width, which is detected at the $6.1 \sigma$ significance level. This confirms the trend previously observed in a much smaller sample by Wolfe \& Prochaska (1998). The best-fit linear relation is $[\mathrm{X} / \mathrm{H}]=1.55( \pm 0.12) \log \Delta V-4.33( \pm 0.23)$, with $\Delta V$ expressed in $\mathrm{km} \mathrm{s}^{-1}$. The slope of the DLA velocity-metallicity relation is the same within uncertainties between the higher $\left(z_{\mathrm{abs}}>2.43\right)$ and the lower $\left(z_{\mathrm{abs}} \leq 2.43\right)$ redshift halves of our sample. However, the two populations of systems are statistically different. There is a strong redshift evolution in the sense that the median metallicity and median velocity width increase with decreasing redshift. We argue that the existence of a DLA velocity-metallicity correlation, over more than a factor of 100 spread in metallicity, is probably the consequence of an underlying mass-metallicity relation for the galaxies responsible for DLA absorption lines. Assuming a simple linear scaling of the galaxy luminosity with the mass of the dark-matter halo, we find that the slope of the DLA velocity-metallicity relation is consistent with that of the luminosity-metallicity relation derived for local galaxies. If the galaxy dynamical mass is indeed the dominant factor setting up the observed DLA velocity-metallicity correlation, then the DLA systems exhibiting the lowest metallicities among the DLA population should, on average, be associated with galaxies of lower masses (e.g., gas-rich dwarf galaxies). In turn, these galaxies should have the lowest luminosities among the DLA galaxy population. This could explain the difficulties of detecting high-redshift DLA galaxies in emission.
\end{abstract}

Key words. galaxies: halos - galaxies: high-redshift - galaxies: ISM - quasars: absorption lines - cosmology: observations

\section{Introduction}

Over the past decade, significant progress in our understanding of early galaxy evolution has been made with large samples of high-redshift galaxies drawn from deep multi-band imaging (Steidel et al. 2003, and references therein). However, even before the first surveys for Lyman-Break Galaxies (LBGs) had begun, samples of DLA absorbers observed on the lines-of-sight to distant quasars had been constructed (Wolfe et al. 1986, 1995). These absorbers were thought at the time to be the best carriers of information on the population of high-redshift galaxies, but, despite many attempts to identify the galaxies responsible for DLA absorption lines (hereafter called DLA galaxies), only very

* Based on data gathered at the European Southern Observatory (ESO) using the Ultraviolet and Visible Echelle Spectrograph (UVES) installed at the Very Large Telescope (VLT), Unit-2, Kueyen, on Cerro Paranal in Chile. few could be detected in emission (see, e.g., Møller \& Warren 1993; Djorgovski et al. 1996; Leibundgut \& Robertson 1999; Fynbo et al. 1999; Møller et al. 2002, 2004; Christensen et al. 2004; Weatherley et al. 2005). However, there is little doubt that DLA systems arise from the densest regions of the Universe and are closely associated with galaxies. It is therefore crucial to establish the connection between the absorption-selected DLA systems and emission-selected galaxies. In addition, the detailed information that becomes available only through the combination of morphology, colour, and luminosity, with QSO absorption-line spectroscopy, makes these galaxy/absorber associations unique laboratories to study the physical processes at work during galaxy formation (see Pei et al. 1999).

Progress in this field has been slow. Firstly, a huge amount of work is needed to derive important parameters in DLA systems such as gas kinematics, metallicity, or dust and molecular fractions (e.g., Pettini et al. 1997; Prochaska \& Wolfe 1997, 1998, 1999; Prochaska et al. 2001; Ledoux et al. 1998, 2003). 
Secondly, as mentioned above, the known high-redshift DLA systems have proved to be very difficult to detect in emission. This has caused some confusion, and for awhile suggestions were put forward that DLA absorbers may not be related to high-redshift galaxies at all. Mo et al. (1998) and Haehnelt et al. (1998b, 2000) resolved this issue showing that the difficulty of detecting high-redshift DLA systems in emission is an unavoidable consequence of the absorption cross-section selection, which tends to reveal faint galaxies because they have an integrated cross-section larger than that of bright galaxies (see also Fynbo et al. 1999).

Recently, Møller et al. (2004) tentatively suggested that, if a galaxy luminosity-metallicity relation similar to that observed at $0 \lesssim z \lesssim 1$ (e.g., Garnett 2002; Kobulnicky et al. 2003; Lamareille et al. 2004; Tremonti et al. 2004) were already in place at high redshifts, then it would be possible to significantly increase the DLA galaxy detection probability by carefully selecting DLA systems with the highest metallicities. In fact, the few DLA galaxies that have to date been identified in emission do give support to the conjecture that a luminosity-metallicity relation was already in place at $z \approx 2-3$, although the result is only marginally statistically significant (Møller et al. 2004). This is in line with the near-solar or even super-solar metallicities derived for bright Lyman-break or bright $K$-band selected galaxies at similar redshifts (Shapley et al. 2004; de Mello et al. 2004). In addition, a mass-metallicity relation has recently been put into evidence for UV-selected star-forming galaxies at $z \sim 2.3$ by Erb et al. (2006).

In this paper, we provide for the first time evidence of the existence of a velocity-metallicity correlation for high-redshift DLA galaxies that could be the consequence of an underlying mass-metallicity relation for the galaxies responsible for DLA absorption lines. From the observation of a sample of 17 DLA systems at $z_{\text {abs }}<3$, Wolfe \& Prochaska (1998) previously showed that the DLA systems exhibiting the largest line profile velocity widths span a narrow range of high metallicities. However, these authors also suggested that systems with small velocity widths span a wide range of metallicities. Recently, Péroux et al. (2003) found a hint of an increase of the mean DLA metallicity with increasing velocity width, but the statistical significance of their result is low. In this paper, we use our database of VLT-UVES quasar spectra to build up a sample of 70 DLA or strong sub-DLA systems with total neutral hydrogen column densities of $\log N(\mathrm{HI}) \gtrsim 20$ and redshifts in the range $1.7<z_{\mathrm{abs}}<4.3$. We present new, homogeneous measurements of DLA metallicities and line profile velocity widths in Sect. 2, and the velocity-metallicity correlation in Sect. 3 . We discuss the use of the DLA gas kinematics as a proxy for the mass of DLA galaxies and the possibility of the existence of a mass-metallicity relation for high- $z$ DLA galaxies in Sect. 4, and we conclude in Sect. 5.

\section{UVES DLA sample}

\subsection{Metallicity measurements}

Most of the systems in our sample were selected from the followup of the Large Bright QSO Survey (LBQS; Wolfe et al. 1995) and observed at the ESO VLT with UVES between 2000 and 2004 in the course of a systematic search for molecular hydrogen at $z_{\text {abs }}>1.8$ (Petitjean et al. 2000; Ledoux et al. 2003). Our total sample comprises 57 DLA systems $(\log N(\mathrm{HI}) \geq 20.3)$ and 13 strong sub-DLA systems with total neutral hydrogen column densities in the range $20.0 \lesssim \log N(\mathrm{HI})<20.3$. This is only slightly lower than the classical definition of DLA systems to ensure that these absorbers are mostly neutral and share the same physical nature as classical DLA systems (see Viegas 1995).

We have carefully measured or remeasured total neutral hydrogen column densities, $\log N(\mathrm{H} \mathrm{I})$, and average DLA metallicities for all the systems in our sample. Results are summarised in Table 1. The absorption line analysis was performed in an homogeneous manner using standard Voigt-profile fitting techniques adopting the oscillator strengths compiled by Morton (2003). For the damping coefficients, we also adopted here the compilation by Morton (2003), which results in some cases in a slight increase of $\log N(\mathrm{HI})$ values compared to Ledoux et al. (2003). Total metal column densities were derived as the sum of the column densities measured in individual components of the line profiles. Average gaseous metallicities relative to solar, $[\mathrm{X} / \mathrm{H}] \equiv \log [N(\mathrm{X}) / N(\mathrm{H})]-\log [N(\mathrm{X}) / N(\mathrm{H})]_{\odot}$, were calculated using solar abundances listed in Morton (2003), which are based on meteoritic data from Grevesse \& Sauval (2002). To avoid problems related to possible depletion onto dust grains, metallicities given in Table 1 were computed for elements that are known to deplete very little in the ISM of the Galaxy. The reference element was taken to be $\mathrm{X}=\mathrm{Zn}$ when $\mathrm{Zn}$ II is detected, or else either S or Si was used (see Ledoux et al. 2003, for a discussion).

A noticeable property of this large dataset is that it samples both ends of the DLA metallicity distribution, from $[\mathrm{X} / \mathrm{H}] \approx$ -2.6 up to about half of solar (see Table 1), well.

\subsection{Low-ionization line kinematics}

For each of the systems, we also determined the velocity widths of metal absorption line profiles. Low-ionization transition lines that are not strongly saturated were selected to trace the kinematics induced predominantly by gravity. For high-ionization lines, the velocity widths could be dominated by peculiar ejections of hot gas. We measured the line profile velocity widths following Prochaska \& Wolfe (1997; see also Haehnelt et al. $1998 b)$. We used the criterion that the residual intensity $I$ of the strongest absorption feature in the selected line profile must satisfy $0.1<I / I_{\mathrm{c}}<0.6$, where $I_{\mathrm{c}}$ is the intensity level of the adjacent continuum. This criterion selects transitions that are neither strongly saturated (in which case the optical depth cannot be derived and the velocity width could be overestimated), nor too weak (in which case the velocity width could be underestimated because part of the gas would be undetected). For the few systems for which none of the observed lines satisfies the above criterion, we used the mean value of the velocity widths calculated from (i) a slightly more saturated line, and (ii) a slightly weaker line than what the criterion specifies (see Table 1). From visual inspection of the strongest low-ionization line profiles of a given system, we established the velocity range over which the previously selected line profile should be integrated (this corresponds to the interval $\left[\lambda_{1} ; \lambda_{2}\right]$ in Fig. 1).

To ease comparison with previous works (e.g., Prochaska \& Wolfe 1997), we then calculated the line profile velocity width, $\Delta V$, as $c[\lambda(95 \%)-\lambda(5 \%)] / \lambda_{0}$, where $\lambda(5 \%)$ and $\lambda(95 \%)$ are the wavelengths corresponding to, respectively, the five per cent and 95 per cent percentiles of the apparent optical depth distribution, and $\lambda_{0}$ is the first moment of this distribution (see Fig. 1). Note that all our spectra have typical signal-to-noise ratios larger than 30. Excluding the extended wings of the line profiles allows us to avoid taking into account satellite components that are not strictly related to the bulk of the systems. 
Table 1. UVES DLA sample: average metallicities and velocity widths of low-ionization line profiles*.

\begin{tabular}{|c|c|c|c|c|c|c|c|c|}
\hline Quasar & Other name & $z_{\mathrm{em}}$ & $z_{\mathrm{abs}}^{1}$ & $\log N(\mathrm{HI})$ & {$[\mathrm{X} / \mathrm{H}]^{2}$} & $\mathrm{X}$ & $\begin{array}{c}\Delta V \\
\left(\mathrm{~km} \mathrm{~s}^{-1}\right)\end{array}$ & $\begin{array}{c}\text { Selected } \\
\text { transition lines }^{3}\end{array}$ \\
\hline Q 0000-263 & LBQS 0000-2619 & 4.11 & 3.390 & $21.40 \pm 0.08$ & $-2.06 \pm 0.09$ & $\mathrm{Zn}$ & 33 & O I $\lambda 950$ \\
\hline Q 0010-002 & LBQS 0010-0012 & 2.15 & 2.025 & $20.95 \pm 0.10$ & $-1.43 \pm 0.11$ & $\mathrm{Zn}$ & 32 & Si II $\lambda 1808$ \\
\hline Q 0013-004 & LBQS 0013-0029 & 2.09 & 1.973 & $20.83 \pm 0.05$ & $-0.59 \pm 0.05$ & $\mathrm{Zn}$ & 720 & Fe II $\lambda 1608$ \\
\hline Q 0058-292 & LBQS 0058-2914 & 3.09 & 2.671 & $21.10 \pm 0.10$ & $-1.53 \pm 0.10$ & $\mathrm{Zn}$ & 34 & Si II $\lambda 1808$ \\
\hline Q 0100+130 & LBQS 0100+1300 & 2.69 & 2.309 & $21.35 \pm 0.08$ & $-1.58 \pm 0.08$ & $\mathrm{Zn}$ & 37 & Cr II $\lambda 2056$ \\
\hline Q 0102-190 & LBQS 0102-1902 & 3.04 & 2.370 & $21.00 \pm 0.08$ & $-1.90 \pm 0.08$ & S & 17 & Fe II $\lambda 2374$ \\
\hline Q 0102-190 & LBQS 0102-1902 & 3.04 & 2.926 & $20.00 \pm 0.10$ & $-1.50 \pm 0.10$ & $\mathrm{Si}$ & 146 & Si II $\lambda 1526$ \\
\hline Q 0112-306 & LBQS 0112-3041 & 2.99 & 2.418 & $20.50 \pm 0.08$ & $-2.42 \pm 0.08$ & $\mathrm{Si}$ & 31 & Si II $\lambda 1526$ \\
\hline Q 0112-306 & LBQS 0112-3041 & 2.99 & 2.702 & $20.30 \pm 0.10$ & $-0.49 \pm 0.11$ & $\mathrm{Si}$ & 218 & Fe II $\lambda 1608$ \\
\hline Q $0112+030$ & LBQS $0112+0300$ & 2.81 & 2.423 & $20.90 \pm 0.10$ & $-1.31 \pm 0.11$ & $\mathrm{~S}$ & 112 & Fe II $\lambda 1608$ \\
\hline Q $0135-273$ & CTS 1005 & 3.21 & 2.107 & $20.30 \pm 0.15$ & $-1.12 \pm 0.16$ & S & $82 / 103$ & Fe II $\lambda 2260 / \mathrm{Fe}$ ІІ $\lambda 2586$ \\
\hline Q 0135-273 & CTS 1005 & 3.21 & 2.800 & $21.00 \pm 0.10$ & $-1.40 \pm 0.10$ & $\mathrm{~S}$ & $65 / 67$ & S II $\lambda 1259 / \mathrm{Fe}$ II $\lambda 1608$ \\
\hline Q $0216+080$ & LBQS $0216+0803$ & 2.99 & 1.769 & $20.30 \pm 0.10$ & $-1.00 \pm 0.12$ & $\mathrm{Zn}$ & 75 & Fe II $\lambda 2374$ \\
\hline Q $0216+080$ & LBQS $0216+0803$ & 2.99 & 2.293 & $20.50 \pm 0.10$ & $-0.70 \pm 0.11$ & $\mathrm{Zn}$ & 104 & Si II $\lambda 1808$ \\
\hline Q 0336-017 & LBQS 0336-0142 & 3.20 & 3.062 & $21.10 \pm 0.10$ & $-1.41 \pm 0.10$ & $\mathrm{Si}$ & 67 & Si II $\lambda 1808$ \\
\hline Q 0347-383 & LBQS 0347-3819 & 3.22 & 3.025 & $20.73 \pm 0.05$ & $-1.17 \pm 0.07$ & $\mathrm{Zn}$ & 93 & Fe II $\lambda 1608$ \\
\hline Q $0405-443$ & CTS 247 & 3.02 & 1.913 & $20.80 \pm 0.10$ & $-1.03 \pm 0.10$ & $\mathrm{Zn}$ & 98 & Si II $\lambda 1808$ \\
\hline Q $0405-443$ & CTS 247 & 3.02 & 2.550 & $21.15 \pm 0.15$ & $-1.36 \pm 0.16$ & $\mathrm{Zn}$ & 165 & Si II $\lambda 1808$ \\
\hline Q $0405-443$ & CTS 247 & 3.02 & 2.595 & $21.05 \pm 0.10$ & $-1.12 \pm 0.10$ & $\mathrm{Zn}$ & 79 & Si II $\lambda 1808$ \\
\hline Q $0405-443$ & CTS 247 & 3.02 & 2.622 & $20.45 \pm 0.10$ & $-2.04 \pm 0.10$ & $\mathrm{Si}$ & 182 & Si II $\lambda 1526$ \\
\hline Q 0450-131 & $\ldots$ & 2.25 & 2.067 & $20.50 \pm 0.07$ & $-1.62 \pm 0.08$ & $\mathrm{~S}$ & 148 & Fe II $\lambda 1608$ \\
\hline Q 0458-020 & LBQS 0458-0203 & 2.29 & 2.040 & $21.70 \pm 0.10$ & $-1.22 \pm 0.10$ & $\mathrm{Zn}$ & 88 & Cr II $\lambda 2056$ \\
\hline Q 0528-250 & LBQS 0528-2505 & 2.77 & 2.141 & $20.98 \pm 0.05$ & $-1.36 \pm 0.06$ & $\mathrm{Zn}$ & 105 & Si II $\lambda 1808$ \\
\hline Q 0528-250 & LBQS 0528-2505 & 2.77 & 2.811 & $21.35 \pm 0.07$ & $-0.91 \pm 0.07$ & $\mathrm{Zn}$ & 304 & S II $\lambda 1253$ \\
\hline Q 0551-366 & .... & 2.32 & 1.962 & $20.70 \pm 0.08$ & $-0.35 \pm 0.08$ & $\mathrm{Zn}$ & 468 & Si II $\lambda 1808$ \\
\hline Q $0841+129$ & $\ldots$ & 2.50 & 1.864 & $21.00 \pm 0.10$ & $-1.51 \pm 0.11$ & $\mathrm{~S}$ & 32 & S II $\lambda 1259$ \\
\hline Q $0841+129$ & $\ldots$ & 2.50 & 2.375 & $21.05 \pm 0.10$ & $-1.59 \pm 0.10$ & $\mathrm{Zn}$ & 37 & Fe II $\lambda 1125$ \\
\hline Q $0841+129$ & $\ldots$ & 2.50 & 2.476 & $20.80 \pm 0.10$ & $-1.60 \pm 0.10$ & $\mathrm{Zn}$ & 30 & S II $\lambda 1259$ \\
\hline Q $0913+072$ & LBQS 0913+0715 & 2.78 & 2.618 & $20.35 \pm 0.10$ & $-2.59 \pm 0.10$ & $\mathrm{Si}$ & 22 & Si II $\lambda 1526$ \\
\hline Q 1036-229 & CTS 460 & 3.13 & 2.778 & $20.93 \pm 0.05$ & $-1.36 \pm 0.05$ & $\mathrm{~S}$ & 80 & Fe II $\lambda 1081$ \\
\hline Q 1108-077 & BRI 1108-0747 & 3.92 & 3.482 & $19.95 \pm 0.07$ & $-1.63 \pm 0.07$ & $\mathrm{Si}$ & 49 & Si II $\lambda 1526$ \\
\hline Q 1108-077 & BRI 1108-0747 & 3.92 & 3.608 & $20.37 \pm 0.07$ & $-1.59 \pm 0.07$ & $\mathrm{Si}$ & 31 & Fe II $\lambda 1608$ \\
\hline Q 1111-152 & CTS 298 & 3.37 & 3.266 & $21.30 \pm 0.05$ & $-1.65 \pm 0.11$ & $\mathrm{Zn}$ & 140 & Si II $\lambda 1020$ \\
\hline Q 1117-134 & BR 1117-1329 & 3.96 & 3.350 & $20.95 \pm 0.10$ & $-1.41 \pm 0.11$ & $\mathrm{Zn}$ & $43 / 45$ & Si II $\lambda 1808 / \mathrm{Fe}$ II $\lambda 1608$ \\
\hline Q 1157+014 & $\ldots$ & 1.99 & 1.944 & $21.80 \pm 0.10$ & $-1.44 \pm 0.10$ & $\mathrm{Zn}$ & 89 & Fe II $\lambda 2260$ \\
\hline Q 1209+093 & LBQS 1209+0919 & 3.30 & 2.584 & $21.40 \pm 0.10$ & $-1.01 \pm 0.10$ & $\mathrm{Zn}$ & 214 & Si II $\lambda 1808$ \\
\hline Q $1210+175$ & LBQS $1210+1731$ & 2.54 & 1.892 & $20.70 \pm 0.08$ & $-0.93 \pm 0.08$ & $\mathrm{~S}$ & 62 & S II $\lambda 1253$ \\
\hline Q $1223+178$ & LBQS $1223+1753$ & 2.94 & 2.466 & $21.40 \pm 0.10$ & $-1.63 \pm 0.10$ & $\mathrm{Zn}$ & 91 & Si II $\lambda 1808$ \\
\hline Q $1232+082$ & LBQS $1232+0815$ & 2.57 & 2.338 & $20.90 \pm 0.08$ & $-1.43 \pm 0.08$ & $\mathrm{~S}$ & 85 & Si II $\lambda 1808$ \\
\hline Q $1331+170$ & $\ldots$. & 2.08 & 1.776 & $21.15 \pm 0.07$ & $-1.28 \pm 0.08$ & $\mathrm{Zn}$ & 75 & Fe II $\lambda 2374$ \\
\hline Q $1337+113$ & LBQS $1337+1121$ & 2.92 & 2.508 & $20.12 \pm 0.05$ & $-1.81 \pm 0.06$ & $\mathrm{Si}$ & 32 & Fe II $\lambda 2344$ \\
\hline Q $1337+113$ & LBQS $1337+1121$ & 2.92 & 2.796 & $21.00 \pm 0.08$ & $-1.86 \pm 0.09$ & $\mathrm{Si}$ & 42 & Fe II $\lambda 1608$ \\
\hline Q 1340-136 & CTS 325 & 3.20 & 3.118 & $20.05 \pm 0.08$ & $-1.42 \pm 0.08$ & $\mathrm{~S}$ & 153 & Fe II $\lambda 1608$ \\
\hline Q 1409+095 & LBQS 1409+0930 & 2.85 & 2.019 & $20.65 \pm 0.10$ & $-1.62 \pm 0.16$ & $\mathrm{Zn}$ & 39 & Fe II $\lambda 2374$ \\
\hline Q 1409+095 & LBQS $1409+0930$ & 2.85 & 2.456 & $20.53 \pm 0.08$ & $-2.06 \pm 0.08$ & $\mathrm{Si}$ & 69 & Si II $\lambda 1526$ \\
\hline Q $1441+276$ & PSS J 1443+2724 & 4.42 & 4.224 & $20.95 \pm 0.10$ & $-0.63 \pm 0.10$ & $\mathrm{~S}$ & 130 & Fe II $\lambda 1081$ \\
\hline Q 1444+014 & LBQS 1444+0126 & 2.21 & 2.087 & $20.25 \pm 0.07$ & $-0.80 \pm 0.09$ & $\mathrm{Zn}$ & 294 & Fe II $\lambda 1608$ \\
\hline Q $1451+123$ & LBQS $1451+1223$ & 3.25 & 2.255 & $20.35 \pm 0.10$ & $-1.07 \pm 0.15$ & $\mathrm{Zn}$ & 103 & Fe II $\lambda 2374$ \\
\hline Q $1451+123$ & LBQS $1451+1223$ & 3.25 & 2.469 & $20.40 \pm 0.10$ & $-2.27 \pm 0.13$ & $\mathrm{Si}$ & 35 & Fe II $\lambda 2382$ \\
\hline Q $1451+123$ & LBQS $1451+1223$ & 3.25 & 3.171 & $20.20 \pm 0.20$ & $-2.10 \pm 0.21$ & $\mathrm{Si}$ & 45 & Si II $\lambda 1526$ \\
\hline Q2059-360 & $\ldots$. & 3.09 & 2.507 & $20.29 \pm 0.07$ & $-1.85 \pm 0.20$ & $\mathrm{~S}$ & 25 & Fe II $\lambda 2344$ \\
\hline Q 2059-360 & $\ldots$ & 3.09 & 3.083 & $20.98 \pm 0.08$ & $-1.77 \pm 0.09$ & $S$ & 44 & Fe II $\lambda 1121$ \\
\hline Q 2116-358 & HES 2116-3550 & 2.34 & 1.996 & $20.10 \pm 0.07$ & $-0.34 \pm 0.11$ & $\mathrm{Zn}$ & 177 & Fe II $\lambda 1608$ \\
\hline
\end{tabular}

\section{The velocity-metallicity relation}

\subsection{Detected correlation}

In Fig. 2, we plot the average metallicity of each of the 70 DLA or strong sub-DLA systems in our sample, $[\mathrm{X} / \mathrm{H}]$, versus the velocity width of their low-ionization line profiles, $\Delta V$, on a logarithmic scale. A positive correlation between the two quantities is detected at the $6.1 \sigma$ significance level using a Kendall rank correlation test. Note that the DLA and strong sub-DLA 
Table 1. continued.

\begin{tabular}{|c|c|c|c|c|c|c|c|c|}
\hline$\overline{\text { Quasar }}$ & Other name & $z_{\mathrm{em}}$ & $z_{\text {abs }}{ }^{1}$ & 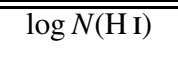 & $\overline{\overline{[\mathrm{X} / \mathrm{H}]^{2}}}$ & $\mathrm{X}$ & $\begin{array}{c}\Delta V \\
\left(\mathrm{~km} \mathrm{~s}^{-1}\right)\end{array}$ & $\begin{array}{c}\text { Selected } \\
\text { transition lines }\end{array}$ \\
\hline Q2138-444 & LBQS 2138-4427 & 3.17 & 2.383 & $20.60 \pm 0.05$ & $-1.15 \pm 0.10$ & $\mathrm{Zn}$ & 131 & Fe II $\lambda 2374$ \\
\hline Q 2138-444 & LBQS 2138-4427 & 3.17 & 2.852 & $20.98 \pm 0.05$ & $-1.74 \pm 0.05$ & $\mathrm{Zn}$ & $58 / 62$ & Si II $\lambda 1808 / \mathrm{Fe}$ II $\lambda 2374$ \\
\hline Q $2152+137$ & PSS J $2155+1358$ & 4.26 & 3.316 & $20.50 \pm 0.15$ & $-1.37 \pm 0.15$ & $\mathrm{Si}$ & 74 & Fe II $\lambda 1608$ \\
\hline Q 2206-199 & LBQS 2206-1958 & 2.56 & 1.921 & $20.67 \pm 0.05$ & $-0.54 \pm 0.05$ & $\mathrm{Zn}$ & 136 & Si II $\lambda 1808$ \\
\hline Q 2206-199 & LBQS 2206-1958 & 2.56 & 2.076 & $20.44 \pm 0.05$ & $-2.32 \pm 0.05$ & $\mathrm{Si}$ & 20 & Si II $\lambda 1304$ \\
\hline Q $2230+025$ & LBQS $2230+0232$ & 2.15 & 1.864 & $20.90 \pm 0.10$ & $-0.81 \pm 0.10$ & $\mathrm{~S}$ & 148 & S II $\lambda 1253$ \\
\hline Q 2231-002 & LBQS 2231-0015 & 3.02 & 2.066 & $20.55 \pm 0.07$ & $-0.65 \pm 0.07$ & $\mathrm{~S}$ & 145 & S II $\lambda 1253$ \\
\hline Q 2243-605 & HES 2243-6031 & 3.01 & 2.331 & $20.65 \pm 0.05$ & $-0.85 \pm 0.05$ & $\mathrm{Zn}$ & 173 & Si II $\lambda 1808$ \\
\hline Q 2332-094 & FIRST J 2334-0908 & 3.32 & 2.287 & $20.07 \pm 0.07$ & $-0.33 \pm 0.07$ & $\mathrm{Zn}$ & 43 & Si II $\lambda 1808$ \\
\hline Q 2332-094 & FIRST J 2334-0908 & 3.32 & 3.057 & $20.50 \pm 0.07$ & $-1.33 \pm 0.08$ & $\mathrm{~S}$ & 111 & Fe II $\lambda 1608$ \\
\hline Q $2343+125$ & .... & 2.51 & 2.431 & $20.40 \pm 0.07$ & $-0.92 \pm 0.07$ & $\mathrm{Zn}$ & 289 & Fe II $\lambda 1608$ \\
\hline Q $2344+125$ & $\ldots$ & 2.76 & 2.538 & $20.50 \pm 0.10$ & $-1.81 \pm 0.10$ & $\mathrm{Si}$ & 69 & Fe II $\lambda 1608$ \\
\hline Q 2348-011 & LBQS 2348-0108 & 3.01 & 2.426 & $20.50 \pm 0.10$ & $-0.64 \pm 0.10$ & $\mathrm{~S}$ & 248 & Fe II $\lambda 1608$ \\
\hline Q 2348-011 & LBQS 2348-0108 & 3.01 & 2.615 & $21.30 \pm 0.08$ & $-2.02 \pm 0.08$ & $\mathrm{Si}$ & $95 / 105$ & Si II $\lambda 1808 /$ Si II $\lambda 1526$ \\
\hline Q 2348-147 & & 2.94 & 2.279 & $20.63 \pm 0.05$ & $-2.10 \pm 0.08$ & $\mathrm{~S}$ & 55 & Fe II $\lambda 2586$ \\
\hline Q 2359-022 & LBQS 2359-0216 & 2.81 & 2.095 & $20.65 \pm 0.10$ & $-0.84 \pm 0.13$ & $\mathrm{Zn}$ & 146 & Fe II $\lambda 1608$ \\
\hline Q 2359-022 & LBQS 2359-0216 & 2.81 & 2.154 & $20.30 \pm 0.10$ & $-1.62 \pm 0.10$ & $\mathrm{Si}$ & 67 & Si II $\lambda 1526$ \\
\hline
\end{tabular}

* Total neutral hydrogen column density and metallicity measurements are from this work, except for the systems at $z_{\text {abs }}=3.390$ toward Q 0000-263 (Molaro et al. 2001), $z_{\mathrm{abs}}=3.025$ and 2.087 toward Q 0347-383 and Q 1444+014 (Ledoux et al. 2003), respectively, $z_{\mathrm{abs}}=1.962$ toward Q 0551-366 (Ledoux et al. 2002), and $z_{\text {abs }}=4.224$ toward Q 1441+276 (Ledoux et al. 2006).

Quoted uncertainties are $1 \sigma$ standard deviations.

${ }^{1}$ There are a few DLA systems with $c \mid z_{\mathrm{em}}-z_{\mathrm{abs}} / /\left(1+z_{\mathrm{abs}}\right)<5000 \mathrm{~km} \mathrm{~s}^{-1}$. These systems were not considered during the correlation analyses. However, their properties (metallicity, $N(\mathrm{H} \mathrm{I})$, ionization level) do not resemble those of the associated systems. They are not associated with the central engine of the quasar, nor ejected by the quasar, but rather associated with dense gas in its surroundings (see text).

${ }^{2}$ Average gaseous metallicities relative to solar, $[\mathrm{X} / \mathrm{H}] \equiv \log [N(\mathrm{X}) / N(\mathrm{H})]-\log [N(\mathrm{X}) / N(\mathrm{H})]_{\odot}$, calculated using solar abundances listed in Morton (2003), which are based on meteoritic data from Grevesse \& Sauval (2002). The reference element was taken to be $\mathrm{X}=\mathrm{Zn}$ when $\mathrm{Zn}$ II is detected, or else either $\mathrm{S}$ or $\mathrm{Si}$ was used.

${ }^{3}$ Transition lines used to determine the velocity widths of low-ionization line profiles. Whenever none of the observed lines satisfied the adopted criterion (see Sect. 2), we used the mean value of the velocity widths calculated from (i) a slightly more saturated line, and (ii) a slightly weaker line than what the criterion specifies.

populations are statistically indistinguishable, even though the mean metallicity is slightly larger among sub-DLA systems. The Kolmogorov-Smirnov test probability that the two velocity width distributions (resp. metallicity distributions) are drawn from the same parent population is $P_{\mathrm{KS}}=0.91$ (resp. $P_{\mathrm{KS}}=$ 0.72 ) in the two-sided case. In the following, we therefore consider the DLA and strong sub-DLA systems together in our sample.

The typical measurement uncertainties in velocity width $( \pm 0.02 \mathrm{dex})$ and metallicity $( \pm 0.10 \mathrm{dex})$ are small compared to the intrinsic scatter of the data points (see Fig. 2). We thus fitted the data using the linear least-square bisector method (Isobe et al. 1990). For the correlation fits, we did not include the DLA systems with $c\left|z_{\mathrm{em}}-z_{\mathrm{abs}}\right| /\left(1+z_{\mathrm{abs}}\right) \leq 5000 \mathrm{~km} \mathrm{~s}^{-1}$, where $z_{\mathrm{em}}$ is the QSO emission redshift. However, these $z_{\mathrm{abs}} \approx z_{\mathrm{em}}$ systems are not associated with the central engine of the quasar, nor ejected by the quasar, but rather associated with dense gas in its surroundings (e.g., Petitjean et al. 1994; Srianand \& Petitjean 2000). It can be seen in Fig. 2 that their inclusion would not affect the observed correlations and that their metallicities and line profile velocity widths are representative of those of the overall DLA population.

The best-fit linear relation is:

$[\mathrm{X} / \mathrm{H}]=1.55( \pm 0.12) \log \Delta V-4.33( \pm 0.23)$,

with $\Delta V$ expressed in $\mathrm{km} \mathrm{s}^{-1}$.

The high-redshift DLA velocity-metallicity correlation presented here is consistent with the results of Nestor et al. (2003) and Turnshek et al. (2005). Using the Sloan Digital Sky Survey (SDSS), these authors found that for strong low-ionization $\mathrm{Mg}$ II absorbers at $1 \lesssim z_{\text {abs }} \lesssim 2$, the $\operatorname{Mg}$ II $\lambda 2796$ equivalent width, or equivalently the absorption line velocity spread, is correlated with the metallicity. In addition, they showed that, within the large equivalent width regime, the average metallicity is larger at lower redshift.

\subsection{Redshift evolution}

The median redshift of our sample is $z_{\text {med }}=2.43$. It can be shown that the two sub-samples of systems with, respectively, $z_{\mathrm{abs}}>2.43$ and $z_{\mathrm{abs}} \leq 2.43$, differ significantly. The median DLA metallicity and median DLA velocity width increase with decreasing redshift: $[\mathrm{X} / \mathrm{H}]=-1.59$ and $\Delta V=69 \mathrm{~km} \mathrm{~s}^{-1}$, and $[\mathrm{X} / \mathrm{H}]=-1.15$ and $\Delta V=92 \mathrm{~km} \mathrm{~s}^{-1}$, in the higher and the lower redshift halves of the sample, respectively. This resembles the point by Wolfe \& Prochaska (1998) that the kinematics and metallicities of the $z_{\mathrm{abs}}>3$ and $z_{\mathrm{abs}}<3$ DLA samples could show significant differences. In addition, a trend for the mean DLA velocity width to increase with decreasing redshift has been reported by Ledoux et al. (1998).

To investigate this behaviour further, we have calculated the projected position, $d_{\text {proj }}$, along the best-fit correlation relation $([\mathrm{X} / \mathrm{H}]$ vs. $\Delta V)$ derived in Sect. 3.1 and drawn as a solid line in Fig. 2, for each system in our sample. We plot the histograms of $d_{\text {proj }}$ for the two redshift sub-samples in Fig. 3. It is apparent that the two histograms differ in the sense that $d_{\text {proj }}$ is larger at lower redshift. This is expected as $d_{\text {proj }}$ increases with increasing velocity width and increasing metallicity. A KolmogorovSmirnov test confirms this: the two populations have only $1 \%$ chance to be drawn from the same parent population. This 

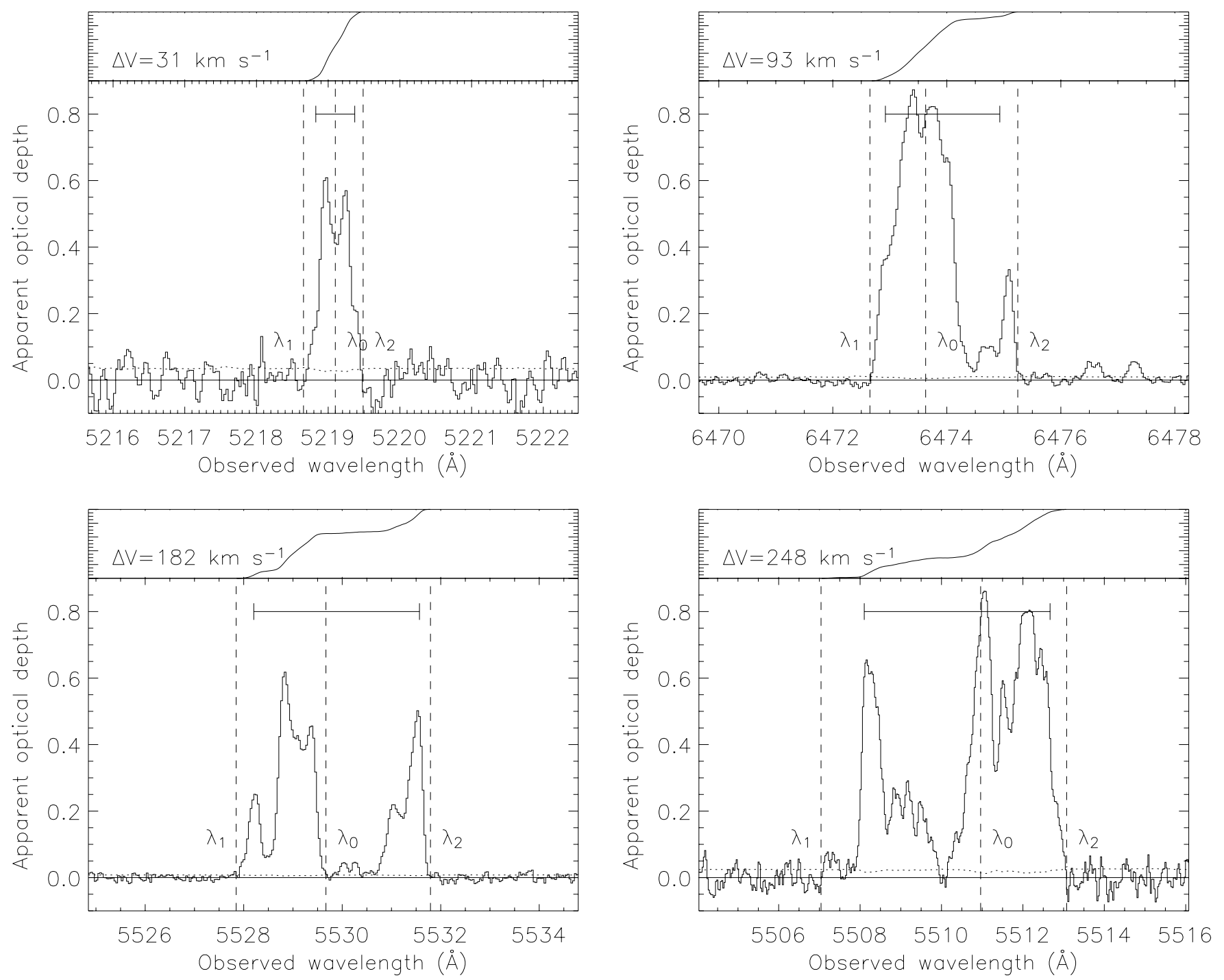

Fig. 1. Measurements of the velocity width of low-ionization line profiles, $\Delta V$. The different panels show the apparent optical depth in the transition line selected for a given system: Si II $\lambda 1526$ at $z_{\text {abs }}=2.418$ toward Q 0112-306 (upper left), Fe II $\lambda 1608$ at $z_{\text {abs }}=3.025$ toward Q $0347-383$ (upper right), Si II $\lambda 1526$ at $z_{\mathrm{abs}}=2.622$ toward Q $0405-443$ (lower left), and Fe II $\lambda 1608$ at $z_{\mathrm{abs}}=2.426$ toward $\mathrm{Q} 2348-011$ (lower right). The top part of each panel shows the integration of the apparent optical depth starting at wavelength $\lambda_{1}$. The horizontal bar below that corresponds to the measurement of $\Delta V$ when $5 \%$ of the total apparent optical depth is avoided at both edges of the profile.

difference is also apparent in Fig. 4, where we plot $[\mathrm{X} / \mathrm{H}]$ versus $\Delta V$ for the two redshift sub-samples separately.

Considering the two redshift sub-samples separately, the best-fit linear relations are:

$[\mathrm{X} / \mathrm{H}]=1.45( \pm 0.17) \log \Delta V-4.01( \pm 0.33)$

for $1.7<z_{\mathrm{abs}} \leq 2.43$, and:

$[\mathrm{X} / \mathrm{H}]=1.47( \pm 0.17) \log \Delta V-4.32( \pm 0.32)$

for $2.43<z_{\mathrm{abs}}<4.3$, with $\Delta V$ expressed in $\mathrm{km} \mathrm{s}^{-1}$.

The significance levels of the correlations in the lower and the higher redshift halves of the sample are $4.6 \sigma$ and $3.9 \sigma$, respectively (see Fig. 4). In addition, the Pearson correlation coefficients are $r=0.72$ and 0.63 , respectively, showing that even in the high-redshift sub-sample, a linear relation is a fairly good description of the data. It is very interesting to note that the correlation relations do not change significantly with redshift, although there is a statistically significant increase in both $[\mathrm{X} / \mathrm{H}]$ and $\Delta V$ with decreasing redshift. This is discussed in Sect. 4.

\subsection{Observational scatter}

Scatter in the data points is expected due to random impact parameters through the absorbing galaxies, and, indeed, the scatter observed in Fig. 2 is much larger than the metallicity measurement uncertainties. Negative radial gradients in metallicity, like those observed in the discs of large nearby spirals, could also contribute to the scatter of the data points. This effect is probably not very important however. For instance, Chen et al. (2005) derived a metallicity gradient of only $-0.041 \pm 0.012$ dex per $\mathrm{kpc}$ from galactic centre to $30 \mathrm{~h}^{-1} \mathrm{kpc}$ radius from a sample of six $z<0.65$ galaxy-DLA pairs. In addition, the magnitude of such gradients in the discs of nearby spirals has recently been questioned (Bresolin et al. 2004).

We note that, in our sample, there are a few systems departing from the general trend. The system at $z_{\mathrm{abs}}=1.973$ toward Q 0013-004 has low-ionization lines extending up to $\sim 1100 \mathrm{~km} \mathrm{~s}^{-1}$ in velocity space (Petitjean et al. 2002), which is a much larger value than for the other systems in our sample. The DLA system at $z_{\mathrm{abs}}=2.622$ toward Q 0405-443 has a 


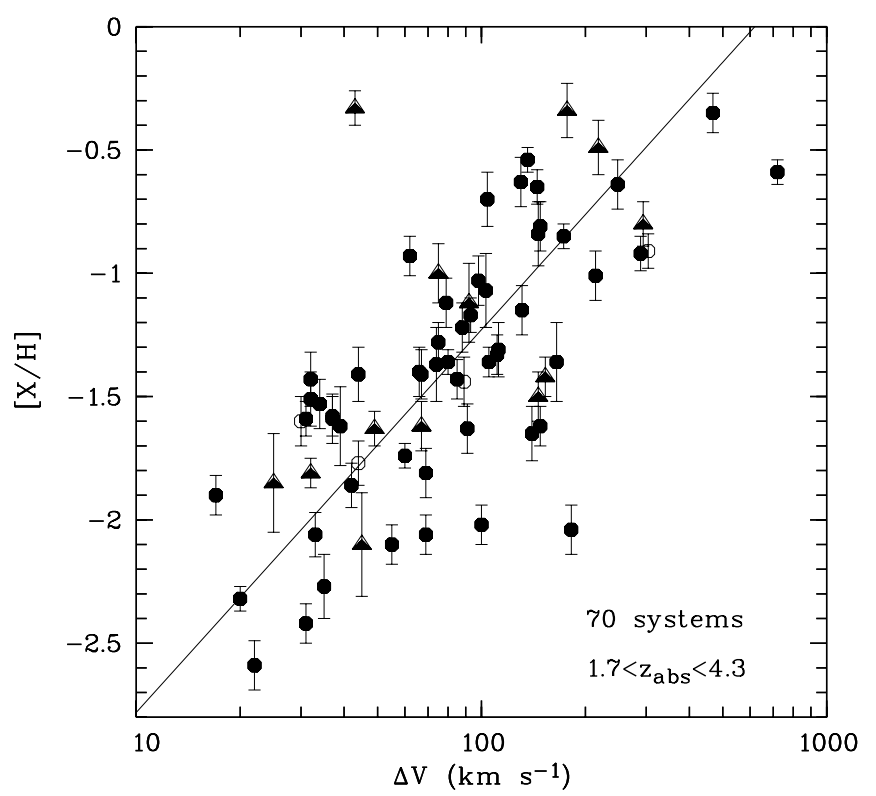

Fig. 2. Average metallicity of each of the 70 DLA or strong subDLA systems in our sample, $[\mathrm{X} / \mathrm{H}]$, vs. the velocity width of their low-ionization line profiles, $\Delta V$, displayed on a logarithmic scale. A positive correlation between the two quantities is detected at the $6.1 \sigma$ significance level using a Kendall rank correlation test. The linear leastsquare bisector fit is shown as a solid line. Sub-DLA systems, with total neutral hydrogen column densities $20 \lesssim \log N(\mathrm{HI})<20.3$, are indicated by filled triangles. There are a few DLA systems with $c\left|z_{\mathrm{em}}-z_{\mathrm{abs}}\right| /\left(1+z_{\mathrm{abs}}\right) \leq 5000 \mathrm{~km} \mathrm{~s}^{-1}$. For the sake of completeness, they are shown here as empty circles, but they were not considered in the analysis.

low metallicity, $[\mathrm{Si} / \mathrm{H}]=-2.04$, and a comparatively large velocity width for this metallicity, $\Delta V=182 \mathrm{~km} \mathrm{~s}^{-1}$ (see Fig. 1, lower left-hand side panel). An inspection of these two cases indicates that the low-ionization line profiles are clumpy, being made of four and two well-separated clumps, respectively. These systems could arise in galaxy groups, tidal streaming in galaxy mergers or the formation of galactic structures (see, e.g., Ledoux et al. 1998; Nulsen et al. 1998; Haehnelt et al. 1998b; Maller et al. 2001). The third peculiar system, the sub-DLA system at $z_{\text {abs }}=2.287$ toward Q 2332-094, is the highest metallicity absorber in our sample with $[\mathrm{Zn} / \mathrm{H}]=-0.33$. It has a surprisingly small velocity width for such a high metallicity, however: its profile is a blend of two sharp metal lines resulting in a velocity width of $\Delta V=43 \mathrm{~km} \mathrm{~s}^{-1}$. These systems are interesting by themselves. However, we note that it is not unexpected to find such deviant cases in a sample that large.

\section{Discussion}

\subsection{Kinematics as a proxy for the mass of DLA galaxies}

The existence of a DLA velocity-metallicity correlation, over more than a factor of 100 spread in metallicity, can be understood as the consequence of an underlying mass-metallicity relation for the galaxies responsible for DLA absorption lines. Peculiar ejection of hot gas should indeed primarily affect the kinematics of high-ionization lines such as C IV and Si IV. Our measurements of line profile velocity widths in DLA systems are based on low-ionization lines, which should instead be dominated by motions on a galactic scale governed, or induced, by gravity.

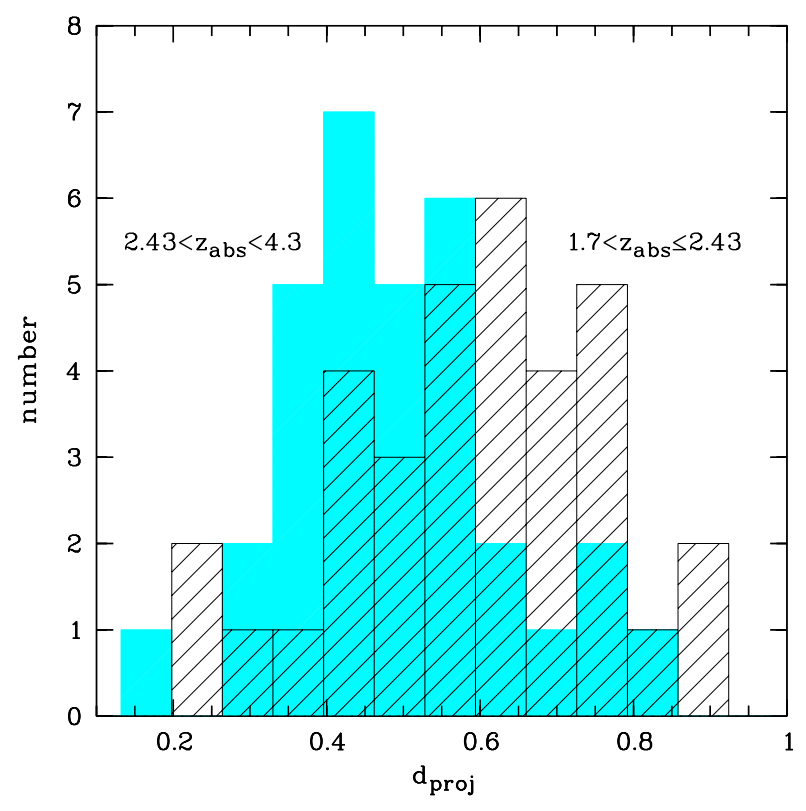

Fig. 3. Histogram of the projected position, $d_{\text {proj }}$, of each system along the best-fit correlation relation plotted as a solid line in Fig. 2, with $d_{\text {proj }}=0$ (resp. $\left.d_{\text {proj }}=1\right)$ at the intersection of this line with the horizontal line corresponding to $[\mathrm{X} / \mathrm{H}]=-3(\mathrm{resp} .[\mathrm{X} / \mathrm{H}]=0)$. The shaded (resp. hashed) histogram represents the higher (resp. lower) redshift half of the sample.

For disc galaxies, the rotation velocity is a direct measure of the galaxy's total mass. Lines-of-sight that do not pass through the centre of the galaxy will not trace the full depth of the potential well and, therefore, will tend to show smaller velocity dispersions than the rotation velocity (see the models by Prochaska \& Wolfe 1997; and Wolfe \& Prochaska 1998). Hence, for random lines-of-sight through a large sample of disc galaxies, there should be a mean relationship between mass and profile velocity width, albeit with a large scatter induced by the range of impact parameters and inclination angles probed by the observations.

The infall/outflow of gas, or merging of galaxy sub-clumps, will also produce kinematically broadened line profiles with velocity widths scaling as the infall/outflow velocities, which again scale as the depth of the combined potential well of the galaxies or mergers. Simulations have shown that in that case, the line profile velocity width is a good indicator of the circular velocity of the underlying dark-matter halo with $\Delta V \sim 0.6 v_{\mathrm{c}}$ (Nulsen et al. 1998; Haehnelt et al. 1998b; Maller et al. 2001). The scatter in this relation is about a factor of two and corresponds approximately to the width of the correlation shown in Fig. 2.

In addition, there is a positive correlation between the projected stellar mass density and the neutral hydrogen column density of DLA systems, and a good correspondence in the spatial distribution of stars and DLA systems in simulations including star formation, supernova feedback, and feedback by galactic winds (Nagamine et al. 2004).

\subsection{Implications and prospects}

For reasons discussed above, we assume here that the dynamical mass of DLA galaxies is the dominant factor setting up the observed DLA velocity-metallicity correlation. 

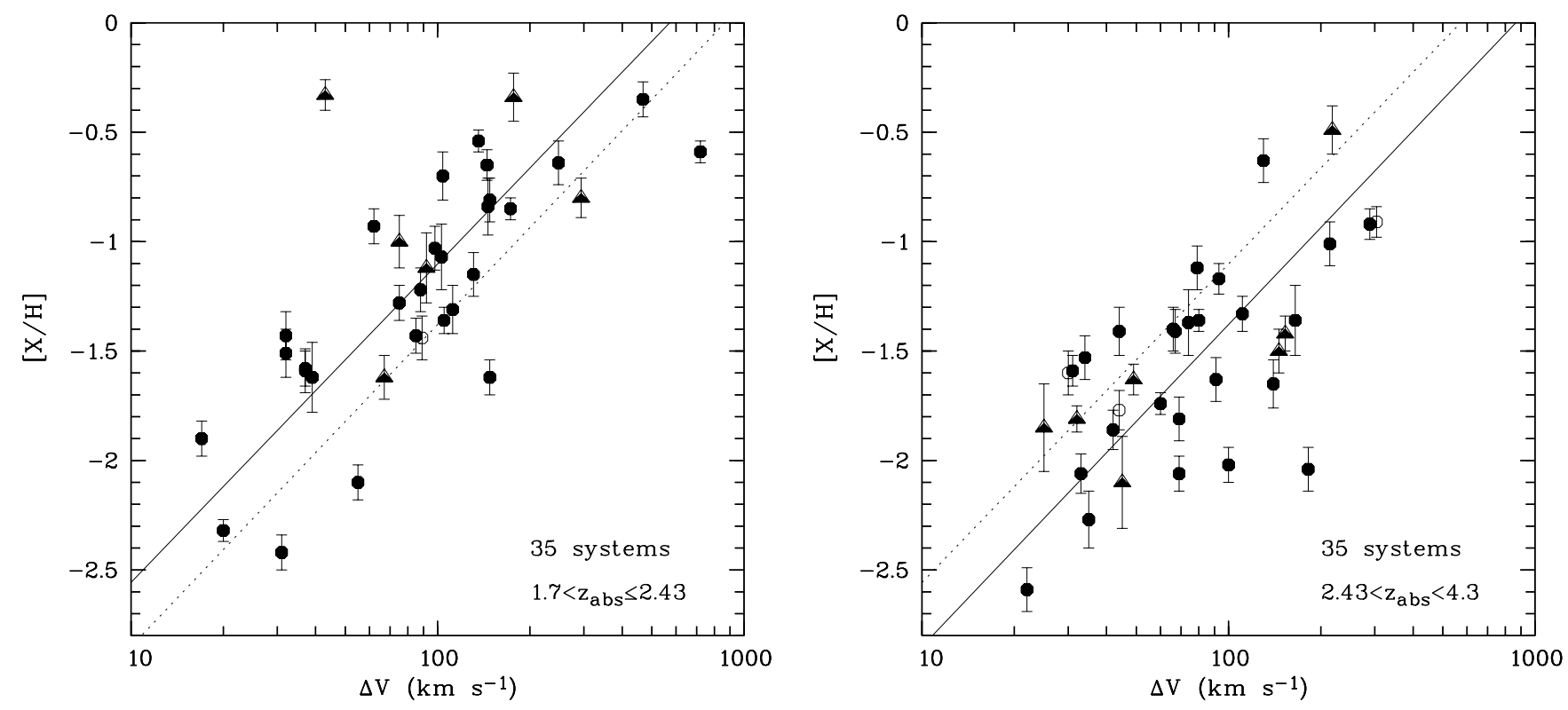

Fig. 4. Same as Fig. 2, but for the sub-sample of systems with $1.7<z_{\text {abs }} \leq 2.43$ (left hand-side panel) or the sub-sample of systems with $2.43<z_{\text {abs }}<4.3$ (right hand-side panel). Positive correlations between the two quantities are detected in both the lower and the higher redshift sub-samples with significance levels of $4.6 \sigma$ and $3.9 \sigma$, respectively. Linear least-square bisector fits to each sub-sample are shown as solid lines in the corresponding panels; the dotted lines are the fits to the other sub-sample. There are a few DLA systems with $c\left|z_{\mathrm{em}}-z_{\mathrm{abs}}\right| /\left(1+z_{\mathrm{abs}}\right) \leq 5000 \mathrm{~km} \mathrm{~s}{ }^{-1}$ in our sample. For the sake of completeness, they are shown here as empty circles, but they were not considered in the analysis.

\subsubsection{Mass-metallicity relation}

The slope of the DLA velocity-metallicity relation is the same within uncertainties for the two redshift halves of our sample (see Sect. 3.2). There is a possible increase of the intercept point of this relation with decreasing redshift (see Fig. 4), but this result is not statistically significant due to the large scatter of the data points around the mean relations (see Eqs. (2) and (3)). However, the two redshift sub-samples differ in the sense that the median DLA metallicity and median DLA velocity width increase with decreasing redshift (see Sect. 3.2 and Fig. 3). This suggests that galaxy halos of a given mass (resp. a given metallicity) are becoming more metal-rich (resp. less massive) with time.

This result is consistent with the work of Savaglio et al. (2005), who proposed a redshift-dependent galaxy massmetallicity relation from the study of $0.4<z<1.0$ galaxies selected from the Gemini Deep Deep Survey and the Canada-France Redshift Survey. We also note that a massmetallicity relation has recently been put into evidence for UV-selected star-forming galaxies at $z \sim 2.3$ (Erb et al. 2006).

\subsubsection{Luminosity-metallicity relation}

From Cold Dark Matter (CDM) simulations, Haehnelt et al. (1998b) have shown that the velocity width of DLA systems, $\Delta V$, can be related statistically to the circular velocity of the underlying dark-matter halo, $v_{\mathrm{c}}=(G M / r)^{1 / 2}$, where $M$ is the mass in a sphere overdense by a factor of 200 compared to the mean cosmic density. They found $\Delta V \sim 0.6 v_{\mathrm{c}}$. According to Haehnelt et al. (1998a, 2000), the luminosity function of $z \sim 3$ galaxies can be reproduced if a simple linear scaling of the luminosity with the mass of the dark-matter halo is assumed, i.e., $m_{R}=-7.5 \log \left(v_{\mathrm{c}} / 200 \mathrm{~km} \mathrm{~s}^{-1}\right)+m_{R}^{0}$, where $m_{R}$ is the galaxy apparent $R$-band magnitude and $m_{R}^{0}=26.6$ for the $\Lambda$-CDM model. Using the best-fit to the velocity-metallicity relation for $1.7<z_{\text {abs }} \leq 2.43$ DLA systems (Eq. (2)), and $z_{\text {med }}=2.09$ for this sub-sample, we derive:

$[\mathrm{X} / \mathrm{H}]=-0.19( \pm 0.02) M_{R}-4.76( \pm 0.42)+0.19( \pm 0.02) K_{R}$

where $M_{R}$ is the galaxy absolute $R$-band magnitude and $K_{R}$ is the $K$-correction in the $R$-band. Using the best-fit to the velocitymetallicity relation for $2.43<z_{\text {abs }}<4.3$ DLA systems (Eq. (3)) leads to a similar result.

It is striking to note that the slope of this DLA luminosity-metallicity relation is consistent with that derived by Tremonti et al. (2004) for the luminosity-metallicity relation for $z \sim 0.1$ galaxies selected from the SDSS, $[\mathrm{O} / \mathrm{H}]=-0.185( \pm 0.001) M_{B}-3.452( \pm 0.018)$. The correction from the $R$-band at high redshift to the $B$-band at low redshift is an additional factor that corresponds to a non-positive $K$ correction (Kinney et al. 1996) in Eq. (4). Therefore, the intercept points of the two luminosity-metallicity relations are different in the sense that galaxies of a given luminosity (resp. a given metallicity) are becoming more metal-rich (resp. fainter) with time.

\section{Conclusions}

Using a sample of 70 DLA or strong sub-DLA systems with total neutral hydrogen column densities of $\log N(\mathrm{HI}) \gtrsim 20$ and redshifts in the range $1.7<z_{\mathrm{abs}}<4.3$, we have shown that there is a correlation between metallicity $([\mathrm{X} / \mathrm{H}])$ and line profile velocity width $(\Delta V)$ at the $6.1 \sigma$ significance level. The bestfit linear relation is $[\mathrm{X} / \mathrm{H}]=1.55( \pm 0.12) \log \Delta V-4.33( \pm 0.23)$, with $\Delta V$ expressed in $\mathrm{km} \mathrm{s}^{-1}$. We argued that the existence of a DLA velocity-metallicity correlation, over more than a factor of 100 spread in metallicity, is probably the consequence of an underlying mass-metallicity relation for the galaxies responsible for DLA absorption lines. Assuming a simple linear scaling of the galaxy luminosity with the mass of the dark-matter halo, 
we found that the slope of the DLA velocity-metallicity relation is consistent with that of the luminosity-metallicity relation derived for local galaxies. If the galaxy dynamical mass is indeed the dominant factor setting up the observed DLA velocitymetallicity correlation, then the DLA systems exhibiting the lowest metallicities among the DLA population should, on average, be associated with galaxies of lower masses.

Equation (4) implies that the more than two orders of magnitude spread in DLA metallicity could reflect a more than ten magnitudes spread in DLA galaxy luminosity. Even though lowmass galaxies, i.e., gas-rich dwarf galaxies, can undergo periods of intense star formation activity and, in this case, have high luminosities in the UV, it is a fact that, on average, they show lower star formation rates than more massive galaxies (Brinchmann et al. 2004; see also Okoshi et al. 2004). This may well explain the difficulty of detecting high-redshift DLA galaxies in emission (e.g., Kulkarni et al. 2000, 2001). Furthermore, the non-detection of $\operatorname{Ly} \alpha$ emission from the galaxies responsible for low-metallicity DLA systems, down to Ly $\alpha$ fluxes fainter than most of the Ly $\alpha$ emitters from the deep survey of Fynbo et al. (2003), could be a consequence of their low masses and their correspondingly, on average, low star formation activity.

A significant fraction of DLA galaxies could be actively forming stars for some period of time, but due to their small masses, the bursts of star formation would not be powerful enough and/or would have life times that are too short. Ly $\alpha$ emission would simply be too faint, or would be too strong for too short of a spell of time, to be detected by the current generation of 8-10 m class telescopes. Conversely, the existence of a DLA mass-metallicity relation can explain the recent, tentative result by Møller et al. (2004) that the few DLA systems with detected Ly $\alpha$ emission have higher than average metallicities. This should be confirmed by additional deep imaging of the fields of QSOs with carefully selected DLA absorbers.

The DLA velocity-metallicity correlation relation studied in this paper also needs to be investigated in the context of new high-resolution smoothed-particle hydrodynamics simulations, including the effects of feedback in a self-consistent manner (see, e.g., Nagamine et al. 2004).

Acknowledgements. We thank Sandra Savaglio for sharing results prior to publication. J.P.U.F. is supported by the Danish Natural Science Research Council (SNF). P.P.J. and R.S. gratefully acknowledge support from the Indo-French Centre for the Promotion of Advanced Research (Centre Franco-Indien pour la Promotion de la Recherche Avancée) under contract No. 3004-3. P.P.J. thanks ESO Vitacura for hospitality during the time part of this work was completed.

\section{References}

Bresolin, F., Garnett, D. R., \& Kennicutt, R. C. 2004, ApJ, 615, 228 Brinchmann, J., Charlot, S., White, S. D. M., et al. 2004, MNRAS, 351, 1151 Chen, H.-W., Kennicutt, R. C., \& Rauch, M. 2005, ApJ, 620, 703 Christensen, L., Sánchez, S. F., Jahnke, K., et al. 2004, A\&A, 417, 487 de Mello, D. F., Daddi, E., Renzini, A., et al. 2004, ApJ, 608, L29 Djorgovski, S. G., Pahre, M. A., Bechtold, J., \& Elston, R. 1996, Nature, 382, 234
Erb, D. K., Shapley, A. E., Pettini, M., et al. 2006, ApJ, 644, 813 Fynbo, J. P. U., Møller, P., \& Warren, S. J. 1999, MNRAS, 305, 849 Fynbo, J. P. U., Ledoux, C., Møller, P., Thomsen, B., \& Burud, I. 2003, A\&A, 407, 147

Garnett, D. R. 2002, ApJ, 581, 1019

Grevesse, N., \& Sauval, A. J. 2002, Adv. Space Res., 30, 3

Haehnelt, M. G., Natarajan, P., \& Rees, M. J. 1998a, MNRAS, 300, 817

Haehnelt, M. G., Steinmetz, M., \& Rauch, M. 1998b, ApJ, 495, 647

Haehnelt, M. G., Steinmetz, M., \& Rauch, M. 2000, ApJ, 534, 594

Isobe, T., Feigelson, E. D., Akritas, M. G., \& Babu, G. J. 1990, ApJ, 364, 104

Kinney, A. L., Calzetti, D., Bohlin, R. C., et al. 1996, ApJ, 467, 38

Kobulnicky, H. A., Willmer, C. N. A., Phillips, A. C., et al. 2003, ApJ, 599, 1006

Kulkarni, V. P., Hill, J. M., Schneider, G., et al. 2000, ApJ, 536, 36

Kulkarni, V. P., Hill, J. M., Schneider, G., et al. 2001, ApJ, 551, 37

Lamareille, F., Mouhcine, M., Contini, T., Lewis, I., \& Maddox, S. 2004, MNRAS, 350, 396

Ledoux, C., Petitjean, P., Bergeron, J., Wampler, E. J., \& Srianand, R. 1998, A\&A, 337, 51

Ledoux, C., Srianand, R., \& Petitjean, P. 2002, A\&A, 392, 781

Ledoux, C., Petitjean, P., \& Srianand, R. 2003, MNRAS, 346, 209

Ledoux, C., Petitjean, P., \& Srianand, R. 2006, ApJ, 640, L25

Leibundgut, B., \& Robertson, J. G. 1999, MNRAS, 303, 711

Maller, A. H., Prochaska, J. X., Somerville, R. S., \& Primack, J. R. 2001, MNRAS, 326, 1475

Mo, H. J., Mao, S., \& White, S. D. M. 1998, MNRAS, 295, 319

Molaro, P., Levshakov, S. A., D’Odorico, S., Bonifacio, P., \& Centurión, M. 2001, ApJ, 549, 90

Møller, P., \& Warren, S. J. 1993, A\&A, 270, 43

Møller, P., Warren, S. J., Fall, S. M., Fynbo, J. P. U., \& Jakobsen, P. 2002, ApJ, 574, 51

Møller, P., Fynbo, J. P. U., \& Fall, S. M. 2004, A\&A, 422, L33

Morton, D. C. 2003, ApJS, 149, 205

Nagamine, K., Springel, V., \& Hernquist, L. 2004, MNRAS, 348, 435

Nestor, D. B., Rao, S. M., Turnshek, D. A., \& Vanden Berk, D. 2003, ApJ, 595, L5

Nulsen, P. E. J., Barcons, X., \& Fabian, A. C. 1998, MNRAS, 301, 168

Okoshi, K., Nagashima, M., Gouda, N., \& Yoshioka, S. 2004, ApJ, 603, 12

Pei, Y. C., Fall, S. M., \& Hauser, M. G. 1999, ApJ, 522, 604

Péroux, C., Dessauges-Zavadsky, M., D’Odorico, S., Kim, T.-S., \& McMahon, R. G. 2003, MNRAS, 345, 480

Petitjean, P., Rauch, M., \& Carswell, R. F. 1994, A\&A, 291, 29

Petitjean, P., Srianand, R., \& Ledoux, C. 2000, A\&A, 364, L26

Petitjean, P., Srianand, R., \& Ledoux, C. 2002, MNRAS, 332, 383

Pettini, M., Smith, L. J., King, D. L., \& Hunstead, R. W. 1997, ApJ, 486, 665

Prochaska, J. X., \& Wolfe, A. M. 1997, ApJ, 487, 73

Prochaska, J. X., \& Wolfe, A. M. 1998, ApJ, 507, 113

Prochaska, J. X., \& Wolfe, A. M. 1999, ApJS, 121, 369

Prochaska, J. X., Gawiser, E., \& Wolfe, A. M. 2001, ApJ, 552, 99

Savaglio, S., Glazebrook, K., Le Borgne, D., et al. 2005, ApJ, 635, 260

Shapley, A. E., Erb, D. K., Pettini, M., Steidel, C. C., \& Adelberger, K. L. 2004, ApJ, 612, 108

Srianand, R., \& Petitjean, P. 2000, A\&A, 357, 414

Steidel, C. C., Adelberger, K. L., Shapley, A. E., et al. 2003, ApJ, 592, 728

Tremonti, C. A., Heckman, T. M., Kauffmann, G., et al. 2004, ApJ, 613, 898

Turnshek, D. A., Rao, S. M., Nestor, D. B., Belfort-Mihalyi, M., \& Quider, A. M. 2005, in Proc. IAU Colloquium No. 199: Probing Galaxies through Quasar Absorption Lines, ed. P. R. Williams, C. Shu, \& B. Ménard, 104

Viegas, S. M. 1995, MNRAS, 276, 268

Weatherley, S. J., Warren, S. J., Møller, P., et al. 2005, MNRAS, 358, 985

Wolfe, A. M., \& Prochaska, J. X. 1998, ApJ, 494, L15

Wolfe, A. M., Turnshek, D. A., Smith, H. E., \& Cohen, R. D. 1986, ApJS, 61, 249

Wolfe, A. M., Lanzetta, K. M., Foltz, C. B., \& Chaffee, F. H. 1995, ApJ, 454, 698 